\title{
Photoluminescence Study of Polycrystalline and Single Crystal Diamond
}

\author{
Dipti Ranjan Mohapatra, Padmnabh Rai, Abha Misra, Powan K. Tyagi, \\ and D.S Misra ${ }^{@}$ \\ Department of physics, Indian Institute of Technology Bombay, Powai, Mumbai-400 076, India
}

\begin{abstract}
Employing photoluminescence (PL) spectroscopy, we have carried out a detailed study of the different luminescence center in chemical-vapordeposited (CVD) single-crystal and poly-crystalline diamond and natural diamonds. In addition to the zero phonon line, the fine structure of the PL emission spectra reveals vibronic bands corresponding to both phonon emission and absorption. At lower temperature, the emission intensity increases and peak position shifts towards higher energy band.
\end{abstract}

\section{INTRODUCTION}

Development of high quality chemical vapor deposited (CVD) diamond films has been a challenge amongst the researcher from last decades. The exceptional properties of natural and synthetic diamond films grown by chemical vapor deposition (CVD) techniques makes it a special material for the electronic, optical, and mechanical applications. In contrast to the natural diamond, the present state of CVD diamond films is highly defective, containing a relatively high concentration of impurities and crystal defects. These defects can induce localized states in the forbidden gap of diamond and deteriorate its electrical and optical properties. It is therefore of interest to study the origin of these impurities and defects so as to enable the synthesis of the defect free diamond films. Various optical methods have been used in order to assess impurities and defects in the diamond films. Amongst these methods, photoluminescence (PL) spectroscopy has been widely used for defect characterization in CVD diamond. Here we describe in details the results of the photoluminescence spectroscopy in CVD and natural diamonds at $77 \mathrm{~K}$ for the analysis of impurities and defects.

\section{EXPERIMENT}

The diamond films used in this study were deposited by the microwave-plasma-assisted CVD technique. For the deposition of the poly-crystalline diamond, the silicon wafers with [100] orientation were used as substrates. The applied microwave power was $1 \mathrm{~kW}$, and the substrate temperature was kept at $850^{\circ} \mathrm{C}$. The flow rate of the methane and hydrogen gases was $2 \mathrm{sccm}$ and $247 \mathrm{sccm}$, respectively. The surface of the substrate was prepared for diamond nucleation by scratching with a $0.2 \mu \mathrm{m}$ diamond powder followed by acid and deionized water cleaning. The single-crystals of CVD diamond were deposited at $1000^{\circ} \mathrm{C}$ with a methane flow of $8 \%$ in balance hydrogen. The substrates for single crystals were (100) oriented natural diamond seeds.

The excitation sources for the PL were $325 \mathrm{~nm}$ He-Cd laser and $514.5 \mathrm{~nm}$ argon- ion lasers, which was focused to $20 \mu \mathrm{m}$ diameter spot on the sample. Low temperature $(77 \mathrm{~K})$ PL spectra were collected using liquid nitrogen in a flow cryostat. 


\section{RESULTS AND DISCUSSION}
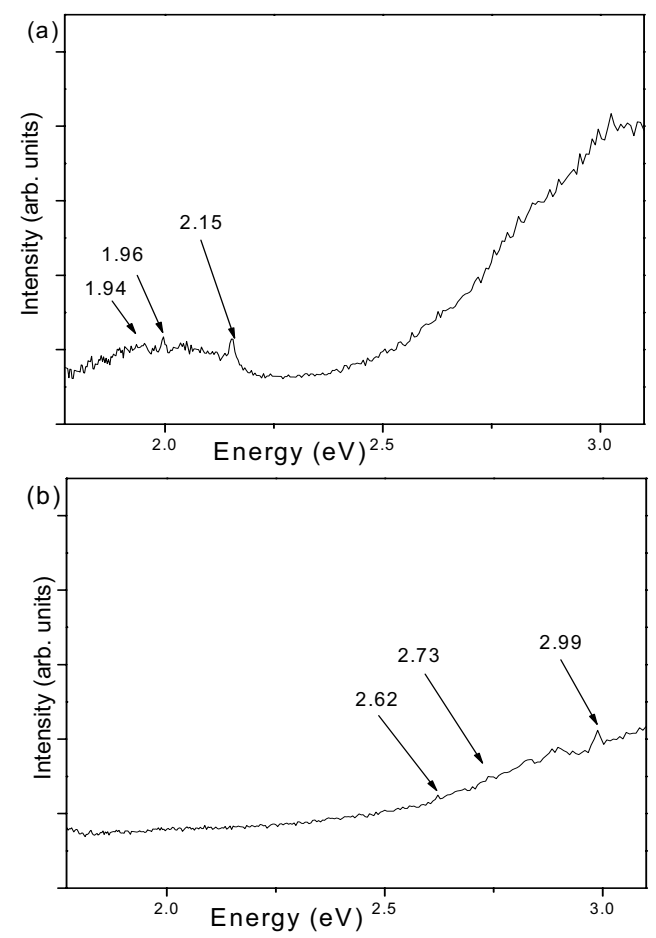

Figure 1(a) and (b) shows the PL spectra of chemical vapor deposited single crystal and natural single crystal diamond excited by $325 \mathrm{~nm}$ laser at room temperature, respectively. Chemical vapor deposited (CVD) single crystal diamond has a strong peak at $2.15 \mathrm{eV}$, which is due to the result of a transition in a center consisting of a single substitutional nitrogen atom with one or more vacancy. The band at $1.94 \mathrm{eV}$ is attributed to the substitutional nitrogen-vacancy $\left([\mathrm{N}-\mathrm{V}]^{-}\right)$center [5], the negative state of the same vacancy observed at $2.15 \mathrm{eV}\left([\mathrm{N}-\mathrm{V}]^{0}\right)$ center. The PL band at $1.96 \mathrm{eV}$ (distinguishable from $1.94 \mathrm{eV}$ band) is might also be due to a nitrogen related center as was observed by Bergman et al. [4]. At higher energy region due to the noise we couldn't able to characterize the energy bands. We need further study to investigate these bands. The natural single crystal diamond shows a zero phonon line (ZPL) at $2.99 \mathrm{eV}$ (shown in Figure 1 (b)) with broad bands around 2.73 and $2.62 \mathrm{eV}$, not observed in CVD single crystal diamond. The zero phonon line at $2.99 \mathrm{eV}$ corresponds to N3 center, which usually observed in natural diamond only [7].

Figure 2 (a) shows low temperature (77K) PL measurements on polycrystalline diamond film deposited on silicon substrate. The spectral position of the zero phonon line (ZPL) at $1.695 \mathrm{eV}$, accompanied by the vibronic side bands at $1.629 \mathrm{eV}$ in the lower frequency region and $1.805 \mathrm{eV}, 1.832$ $\mathrm{eV}, 1.897 \mathrm{eV}$ in higher frequency region.

The vibronic peaks on the low energy side of the zero-phonon line are associated with phonon emission and that of high-energy side is due to the phonon absorption. The origin for the $1.695 \mathrm{eV}$ centre (corresponding to $1.685 \mathrm{eV}$ in room temperature) may be due to the etching of the silicon substrate by high reactive hydrogen plasma.

Figure 1. PL spectra of CVD single crystal diamond and (b) natural single crystal diamond, excited by 325 $\mathrm{nm} \mathrm{He}-\mathrm{CD}$ laser at room temperature.

Silicon atoms are released by the substrate and incorporated into the diamond matrix during the early stages of the growth process. The etching may also depend on the nucleation state of the diamond. The $1.954 \mathrm{eV}$ center (shifted from original $1.945 \mathrm{eV}$ spectral line) was thoroughly studied and has been attributed to the negatively charged nitrogen-vacancy defect $\left([\mathrm{N}-\mathrm{V}]^{-}\right)$as was discussed earlier for single crystal CVD diamond at room temperature, and 2.173 $\mathrm{eV}$ center is attributed to neutral state of the same defect $\left([\mathrm{N}-\mathrm{V}]^{\circ}\right.$ ) (shifted from original $2.154 \mathrm{eV}$ spectral line). The band at $1.963 \mathrm{eV}$ is same as the peak observed in case of single crystal CVD diamond at room temperature. 

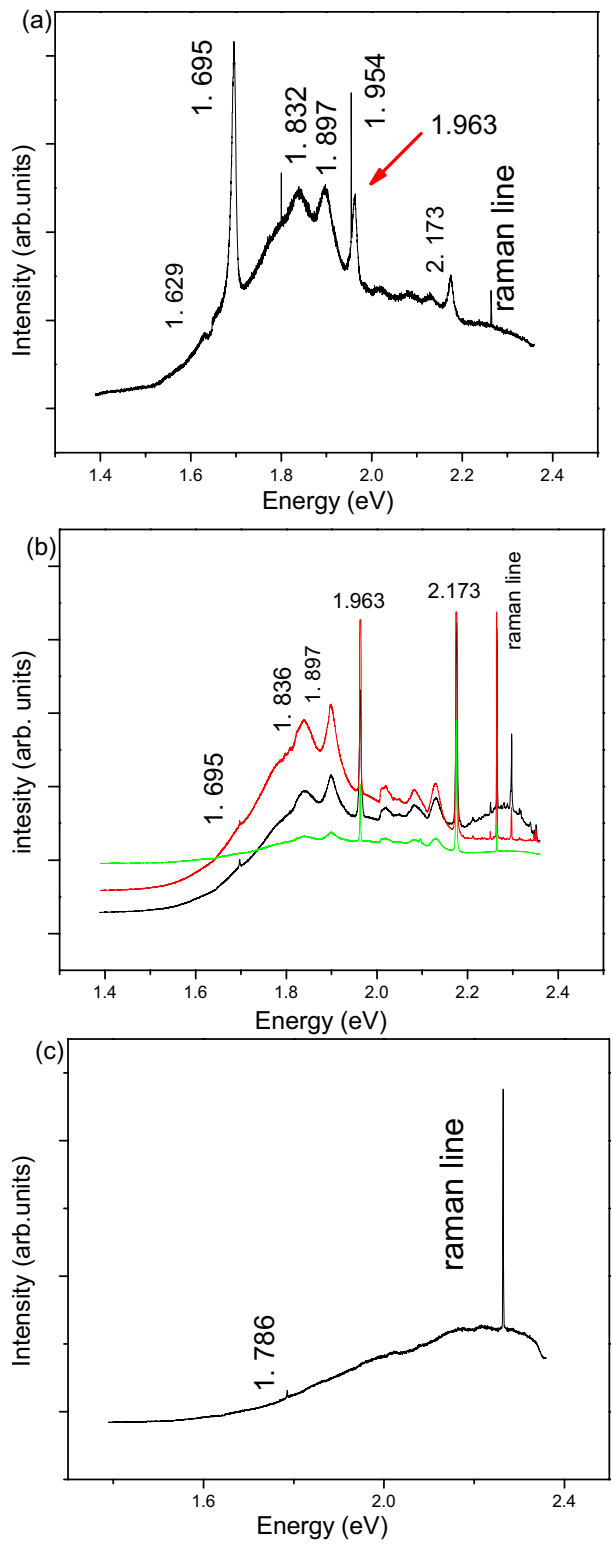

Figure 2. Low temperature $(77 \mathrm{~K})$ micro-PL spectra (a) polycrystalline diamond, (b) three CVD single crystal diamond and (c) natural single crystal diamond, excited by $514.5 \mathrm{~nm} \mathrm{Ar}^{+}$ion laser.

Figure 2 (b) shows the PL spectra of the three single crystal diamonds recorded at $77 \mathrm{~K}$. It is evident that, the luminescence centers, observed at room temperature with $\mathrm{He}-\mathrm{Cd}$ laser are appearing here with greater intensity. The intensity of the PL band at $1.695 \mathrm{eV}$ is comparatively smaller than the band observed in polycrystalline diamond, indicating a small amount of silicon impurity in single crystal diamond. Unlike polycrystalline diamond, the silicon impurity in micro wave plasma chemical vapor (MPCVD) deposited single crystal diamond may be coming from the quartz window used in the growth system. A broad band in the range $2.0 \mathrm{eV}$ to $2.1 \mathrm{eV}$ can be observed. This broad band believed to be due to the phonon interaction with the nitrogen center. In contrast, the PL spectra of a natural diamond at $77 \mathrm{~K}$ (shown in Figure 2 (c)) doesn't show any known nitrogen and silicon impurities. The small band observed at $1.786 \mathrm{eV}$ may be nitrogen related. To analyze this band more experimental work has to be done.

\section{CONCLUSIONS}

In summary, the temperature dependence of photoluminescence (PL) spectra of chemical vapor deposited (CVD) single crystal and polycrystalline diamond films were investigated and compared to the spectra of natural single crystal diamond.

All CVD grown samples exhibit nitrogen and silicon related absorptions, which were absent in the natural diamond. The silicon impurity in polycrystalline diamond is more as comparison to single crystal diamond. In single crystal CVD diamond the silicon impurity comes from the growth system where as etching of silicon substrate cause large silicon impurity in polycrystalline diamond. The low temperature PL spectra were more intense and shifted towards higher energy band.

\section{REFERENCES}

[1] K. Iakoubovskii, G.J. Adriaenssens, Y.K. Vohra, Diamond Related Materials 10 (2001) 485-489.

[2] M.L Fish, O. Massler, J. A. Reid, Diamond Related Materials 8 (1999) 1511-1514

[3] T. Feng, B.D. Schwartz, J. Appl. Phys. 73 (1993) 1415

[4] L. Bergman, M.T. McClure, J. T. Glass, R.J. Nemanichi , J. Appl. Phys. 76 (1994) 3020

[5] J. Walker, Rep. Prog. Phys. 42 (1979) 1606

[6] M.G. Donoto, G. Faggio, G. Messina, Diamond Related Materials 13 (2004) 923-928.

[7] K. Iakoubovskii, G. J. Adriaenssens, Diam. Relat. Mater. 11 (2002) 125-131. 